\title{
BMJ Open Adapting data collection methods in the Australian Life Histories and Health Survey: a retrospective life course study
}

\author{
Hal Kendig, ${ }^{1,2}$ Julie E Byles, ${ }^{1,3}$ Kate O'Loughlin,, ${ }^{1,4}$ James Y Nazroo, ${ }^{1,5}$ \\ Gita Mishra, ${ }^{6}$ Jack Noone, ${ }^{4}$ Vanessa Loh, ${ }^{1,4}$ Peta M Forder ${ }^{3}$
}

To cite: Kendig $\mathrm{H}$, Byles JE, O'Loughlin K, et al. Adapting data collection methods in the Australian Life Histories and Health Survey: a retrospective life course study. BMJ Open 2014;4: e004476. doi:10.1136/ bmjopen-2013-004476

- Prepublication history for this paper is available online To view these files please visit the journal online (http://dx.doi.org/10.1136/ bmjopen-2013-004476)

Received 14 November 2013 Revised 25 February 2014 Accepted 26 February 2014

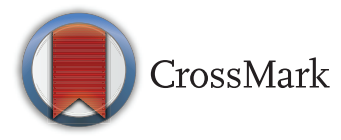

For numbered affiliations see end of article.

Correspondence to Professor Hal Kendig; hal.kendig@anu.edu.au

\section{ABSTRACT}

Objective: Ideally, life course data are collected prospectively through an ongoing longitudinal study. We report adaptive multimethod fieldwork procedures that gathered life history data by mail survey and telephone interview, comparable with the face-to-face methods employed in the English Longitudinal Study on Ageing (ELSA).

Design: The Australian Life Histories and Health (LHH) Survey was a substudy of the Australian 45 and Up Study, with data collection methods modified from the ELSA Study. A self-complete questionnaire and life history calendar were completed by the participants, followed by a computer-assisted telephone interview recording key life events.

Results: The LHH survey developed and tested procedures and instruments that gathered rich life history data within an ongoing Australian Iongitudinal survey on ageing. Data collection proved to be economical. The use of a self-complete questionnaire in conjunction with a life history calendar and coordinated computer-assisted telephone interview was successful in collecting retrospective life course information, in terms of being thorough, practical and efficient. This study has a diverse collection of data covering the life course, starting with early life experiences and continuing with socioeconomic and health exposures and outcomes during adult life.

Conclusions: Mail and telephone methodology can accurately and economically add a life history dimension to an ongoing longitudinal survey. The method is particularly valuable for surveying widely dispersed populations. The results will facilitate understanding of the social determinants of health by gathering data on earlier life exposures as well as comparative data across geographical and societal contexts.

\section{INTRODUCTION}

Governments in many countries are now focusing on how the postwar baby-boom cohort will age relative to earlier cohorts, given the different experiences they will bring to later life. Globally, there is an increasing attention directed towards understanding the impact of life-long socioeconomic

\section{Strengths and limitations of this study}

- This study adapted traditionally resource-intensive data collection methods to more economically collect comprehensive life course data from geographically dispersed participants by utilising a combination of a self-complete questionnaire, a life history calendar and a computer-assisted telephone interview.

- Although the Life Histories and Health Survey collected life history data retrospectively, the use of a life history calendar to aid recall enabled the collection of detailed life history data that would not otherwise have been collected for this early cohort of Australian baby boomers.

- This study provides a unique opportunity to examine the influence of social structures and policy frameworks around health and well-being, allowing for comparisons between those who spent their entire lives in Australia and England, and English migrants to Australia. The study will investigate gender differences over the life course with respect to chronic disease, mental health and socioeconomic position.

- This study may not be representative of all Australians (sampled from the state of New South Wales only). However, the data collected reveal a good variation in each of the study variables with differing experiences reported for lifelong health and family structure across varying socioeconomic circumstances.

- Overall, this study can contribute to our knowledge of the role of earlier life events on health and well-being outcomes for the early cohort of baby boomers in Australia.

opportunities on the resources and vulnerabilities accumulated over the life course to help identify effective points and strategies of intervention. ${ }^{1}$ This growing policy interest in the social determinants of health underscores the importance of societal factors in health and well-being and the ways in which crossnational comparisons can shed light on them. $^{2}$ 
The associations between health and socioeconomic status (SES) are widely known. ${ }^{3-6}$ Life course research suggests that social and environmental disadvantage in childhood $^{7-9}$ and midlife ${ }^{10}$ may be associated with continuing health inequalities in later life. Looking beyond health outcomes, a cross-sectional study of older Australians found that former occupation, education, income and home ownership were independently related to physical and social activity. ${ }^{11}$ Examining life course data may illuminate the causal factors underlying cross-sectional associations between social determinants and life outcomes. The life course research framework provides the capacity to examine the relationships between individual life experiences and changing social structures, and subsequent influences on health, wellbeing and productivity in later life. ${ }^{12}$ The pathways taken by individuals through life intersect with historical time and place to provide a framework for analysing the 'longitudinal accumulation' of transitions and events and their impacts on later life circumstances. ${ }^{8}$

The ideal method for collecting life course data is through a prospective longitudinal study using repeated measures across time. ${ }^{13}$ However, these methods cannot be applied when the cohort of interest is already older and there is no readily available source of retrospective data. In such life course studies, retrospective data are collected to obtain detailed information across multiple domains and events. ${ }^{14}$ Although collected at one point in time, these data can be scheduled across different time periods to simulate multiple observations that allow analysis of the coincidence and scheduling of timevarying covariates, and the modelling of transitions and trajectories in social and health states. ${ }^{15}$ Life history calendars (also known as life event or life grid calendars) have been shown to be effective in reducing recall bias and improving data quality in retrospective studies by providing a graphical time line that helps participants to anchor their responses in relation to different life stages and events. ${ }^{14}{ }^{16-18}$ Such calendars have been used in the German Life History Study, ${ }^{19}$ the Panel Study of Income Dynamics in the USA, ${ }^{20}$ as well as the English Longitudinal Study on Ageing (ELSA), ${ }^{21}$ which was also used as the model for this study.

The Australian Life Histories and Health (LHH) Survey was based on two major ongoing cohort studies: the Sax Institute's Australian ' 45 and Up Study' and 'ELSA'. The LHH Survey was conducted as a substudy within the 45 and Up Study. Using Australia's national public health insurance database (Medicare), the 45 and Up Study recruited over 260000 residents aged 45 years and older from the state of New South Wales (NSW), Australia. The study participants were invited to self-complete a questionnaire collecting demographic, lifestyle and health-related data, and provide consent for follow-up and linkage to a range of health databases. The methods and study sample have been described elsewhere. ${ }^{22}$ ELSA is a multidisciplinary cohort study that has collected information on the mental and physical health and socioeconomic circumstances for a representative national sample of English people aged 50 years and over since 2002. ${ }^{23}$ The advancement of ELSA's life history module was based on extensive work concerning the nature of memory and recall, supporting the use of life history calendars to collect accurate retrospective data. ${ }^{16} 24$

Life history calendars were used in ELSA in conjunction with face-to-face computer-assisted personal interviews (CAPI). While CAPI techniques for survey data collection are seen as the gold standard for research, they are resource-intensive and costly. In contrast, data collection using computer-assisted telephone interviews (CATI) require fewer resources, but remain relatively untested within a life course history framework. Face-to-face data collection was not a viable option in our study due to the geographical dispersion of study participants and cost inefficiencies. We adapted the ELSA data collection method by using life history calendars with CATI and the addition of a self-complete questionnaire. Previous research has shown that using life history calendars in CATI produce more accurate retrospective reports than conventional questionnaires. ${ }^{25}$

The LHH Survey aimed to collect retrospective data on social and health circumstances from birth and throughout adulthood to better understand how socioeconomic and individual circumstances across the life course and changing social structures might affect health, well-being and productivity outcomes in late middle age. This article describes the adaptation of ELSA's CAPI data collection procedures through the combined use of a self-complete questionnaire and CATI utilising a life history calendar as a memory aid.

\section{METHODS}

The first objective was to redesign data collection from the CAPI-only mode to a questionnaire-plus-CATI mode. This approach was taken to reduce the participant's time spent on the telephone, to allow the participants to answer some questions in their own time as well as reducing financial and logistic costs for developing and delivering lengthy telephone interviews. The second objective was to adapt ELSA's life history interview questions for the Australian sample by removing or translating culturally or historically unsuitable items. The third objective was to develop a pen-and-paper life history calendar, which initially covered work, family, health and accommodation histories. As in the ELSA life history interview, LHH participants filled out the life grid in preparation for the follow-up interview. Some of the reminder events used in the ELSA life history calendar were adapted for the Australian context (see figure 1 for an example page from the LHH life history calendar). ${ }^{26}$

\section{Feasibility, pre-test and pilot studies}

Testing of the study procedures began with a feasibility study in 2008 and was refined through a pretest study in 
Early Childhood

\begin{tabular}{|c|c|c|c|c|c|c|c|c|c|c|c|}
\hline & & 1946 & 1947 & $\begin{array}{c}1948 \\
\text { Release of }\end{array}$ & 1949 & $\begin{array}{c}1950 \\
\text { Korean war }\end{array}$ & 1951 & $\begin{array}{c}1952 \\
\text { First atomic }\end{array}$ & 1953 & $\begin{array}{l}1954 \\
\text { Queen }\end{array}$ & 1955 \\
\hline & you date life happenings & & & $\begin{array}{l}\text { Releasse or } \\
\text { Holden car }\end{array}$ & & 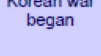 & & $\begin{array}{l}\text { Tests } \\
\text { tests } \\
\text { Australia }\end{array}$ & & $\begin{array}{l}\text { Eueen } \\
\text { Elizabeth } \\
\text { coronation }\end{array}$ & \\
\hline Birth & $\begin{array}{l}\text { Tick the box for the year of } \\
\text { your birth }\end{array}$ & & & & & & & & & & \\
\hline Accommodation & $\begin{array}{l}\text { For each dwelling where } \\
\text { you have lived for } 6 \text { or } \\
\text { more months, write the } \\
\text { suburb (or postcode) into } \\
\text { the year when you moved } \\
\text { there }\end{array}$ & & & & & & & & & & \\
\hline
\end{tabular}

\begin{tabular}{|c|c|c|c|c|c|c|c|c|c|c|c|}
\hline & & 1956 & 1957 & 1958 & 1959 & 1960 & 1961 & 1962 & 1963 & 1964 & 1965 \\
\hline & $\begin{array}{l}\text { Major events that may help } \\
\text { you date life happenings }\end{array}$ & $\begin{array}{l}\text { Melboume } \\
\text { Olympics }\end{array}$ & $\begin{array}{c}\text { Sputnik } \\
\text { orbits }\end{array}$ & $\begin{array}{l}\text { Film South } \\
\text { Pacific } \\
\text { released }\end{array}$ & $\begin{array}{l}\text { Building of } \\
\text { Sydney } \\
\text { Opera } \\
\text { House }\end{array}$ & & $\begin{array}{c}\text { West Side } \\
\text { Story } \\
\text { released }\end{array}$ & & $\begin{array}{c}\text { Cuban } \\
\text { missile crisis }\end{array}$ & $\begin{array}{l}\text { JFK shot } \\
\text { Beatles tour } \\
\text { Aust }\end{array}$ & $\begin{array}{l}\text { Aust. troops } \\
\text { sent to } \\
\text { Vietnam }\end{array}$ \\
\hline Accommodation & $\begin{array}{l}\text { For each dwelling where } \\
\text { you have lived for } 6 \text { or } \\
\text { more months, write the } \\
\text { suburb (or postcode) into } \\
\text { the year when you moved } \\
\text { there }\end{array}$ & & & & & & & & & & \\
\hline \begin{tabular}{|l} 
Partners \\
\end{tabular} & \begin{tabular}{|l|} 
Marriage/de facto \\
relationships [write \\
Partner1, Partner 2 etc in \\
year when you started \\
living together]
\end{tabular} & & & & & & & & & & \\
\hline \begin{tabular}{|l|} 
Children \\
\end{tabular} & $\begin{array}{l}\text { Year of birth of your child } \\
\text { [write Child } 1 \text {, Child } 2 \text { etc] }\end{array}$ & & & & & & & & & & \\
\hline Work & $\begin{array}{l}\text { Jobs: year when you } \\
\text { started each job [write job } \\
\text { title] }\end{array}$ & & & & & & & & & & \\
\hline
\end{tabular}

Figure 1 Example page from the life history calendar used in the, Life Histories and Health Survey.

2010 and a pilot study in early 2011 . The feasibility study was conducted to investigate the viability of collecting life course data through a combination of self-complete questionnaire and a telephone interview. A key objective in developing the study protocol was to achieve a balance between the time needed to gather extensive life history and health information and minimising the participant's fatigue during the telephone interview. The participants completed the questionnaire first and were then requested to fill in the life history calendar prior to the telephone interview. During the interview, the participants were asked to use the life history calendar as a memory aid when reporting their history information. The telephone interview was guided by structured questions and reflected the content requested in the life history calendar.

Following the feasibility study, the data collection instruments were revised and used in a pretest in 2010 to formally evaluate the CATI implementation. Further modifications were made to the data collection instruments and survey process before the pilot study was conducted in 2011. Table 1 summarises the aims and procedures for the feasibility, pretest and pilot studies, outlining the key decisions that were made at each step in the development of the survey protocol. The selfcomplete questionnaire, life history calendar and CATI remained unchanged after the pilot study.

\section{Main study}

\section{Participants}

People were eligible to complete the LHH Survey if they were born between 1947 and 1951 (ie, baby boomers aged 60-64 in 2011), and had completed the baseline 45 and Up questionnaire in 2008, but had not taken part in a prior substudy. Based on the pilot study response rate, 2800 eligible people from the 45 and Up Study were invited to participate (stratified by gender and UK migrant status, see figure 2).

\section{Procedures}

Data were collected from October 2011 to February 2012. Survey packs containing a participant information sheet, consent form, self-complete questionnaire, life history calendar and a postpaid reply envelope were sent by an external organisation specialising in postal surveys. The first mail-out wave included 1600 invitees, with a subsequent mail-out 2 weeks later for the remaining 1200 invitees. A reminder postcard was sent to non-responders 3 weeks after the initial mail-out and a replacement questionnaire was sent 3 weeks after the reminder postcard. Completed questionnaires and consent forms were returned by 1508 participants with an additional 70 invitation packs received marked 'Return to sender'.

The final set of 1261 LHH participants provided consent to participate, sent back a self-completed 


\section{Major aims} Feasibility study (2008)

To investigate the viability of adapting the ELSA life history face-to-face interview for administration to an Australian population using structured telephone interviews as a bridge to the planned use of CATI Sample age and Members (aged 50 years and up) of National source Seniors Australia, Parramatta branch, NSW, Australia

Invitations sent/ respondents/ response rate Materials
Major findings

Changes to the survey protocol
Consent form, participant information statement, introduction letter, life grid calendar, self-completion questionnaire and a return envelope

Snowballing techniques were used to recruit the participants.

Participants returned the consent form and the self-completion questionnaire and were asked to make notes on and retain the life grid calendar for the follow-up telephone interview to be performed by University of Sydney Researchers. The interview process was conducted in two stages. During the initial development stage, the interview questions were modified after each interview based on issues that were identified in the interview. When the interview had reached a stage where most of the participants could complete it in approximately $1 \mathrm{~h}$, the interview format was fixed and the structured interviews were

A pen-and-paper version of the life grid and follow-up telephone interview was an effective means for recording life history information

Following the feasibility study, the CATI protocol was further refined to reduce the length of the telephone interview.

Items that were not deemed central to the Project

were also removed from the self-complete questionnaire

\section{Pretest study (2010)}

To test a refined version of the telephone interview in a formal CATI environment

60-64 years old registered members of the HMRI, Australia

$120 / 24 / 20 \%$

As per feasibility study conducted
One hundred and twenty registered members of the HMRI were invited to take part in the pilot study via an email from the Institute's

administrator. Interested members (32) were sent a survey pack as per the feasibility study A University of Sydney researcher received the

Consent forms and self-complete questionnaire.

The participants' contact details were then passed onto a professional CATI company.

A University of Sydney researcher working on the $\mathrm{LHH}$ project briefed the interviewers. The interviewers were asked to note down any problems the participants had with the interview and to identify ways that the interview could be streamlined

The interviews took, on average, $45 \mathrm{~min}$ to complete. However, the two participants who had not completed the grid took 90 min to relay their life history information to the interviewers A decision was made to not attempt the follow-up interview if the participant had not completed their life grid. In these future instances, a new interview time was to be scheduled.

Life history questions on children were shifted from the CATI to the self-complete and the CATI protocol was further refined to reduce the interview time to an expected $30 \mathrm{~min}$

\section{Pilot study (2011)}

Full test of fieldwork procedures, survey protocol and sampling framework for the main study

60-64 years old participants from the 45 and Up Study, NSW, Australia

$100 / 34 / 34 \%$

As per feasibility study.

The consent form, introduction letter and self-complete questionnaire were combined into a single booklet

Eligible participants randomly selected from the 45 and Up Study participant pool. Survey packs were mailed on behalf of and received by the 45 and Up study. The participants' details were then forwarded to the professional CATI company for the follow-up telephone interview. The participants were contacted within 10 days of receiving their contact information

Interviewers were extensively trained and audited by the University of Sydney researcher on two occasions.

Sampling for English migrants was based on the proportion of UK born participants in the 45 and Up sample aged 60-64 years

Completion of the life grid prior to the interview was a crucial component to successfully gathering life history information within the expected $30 \mathrm{~min}$

The rescheduling of appointments for

participants who had not completed or could not find their life grid was formally noted in the interviewers' manual.

No further changes were made to the study protocol except for some minor items that were added to the self-complete questionnaire 


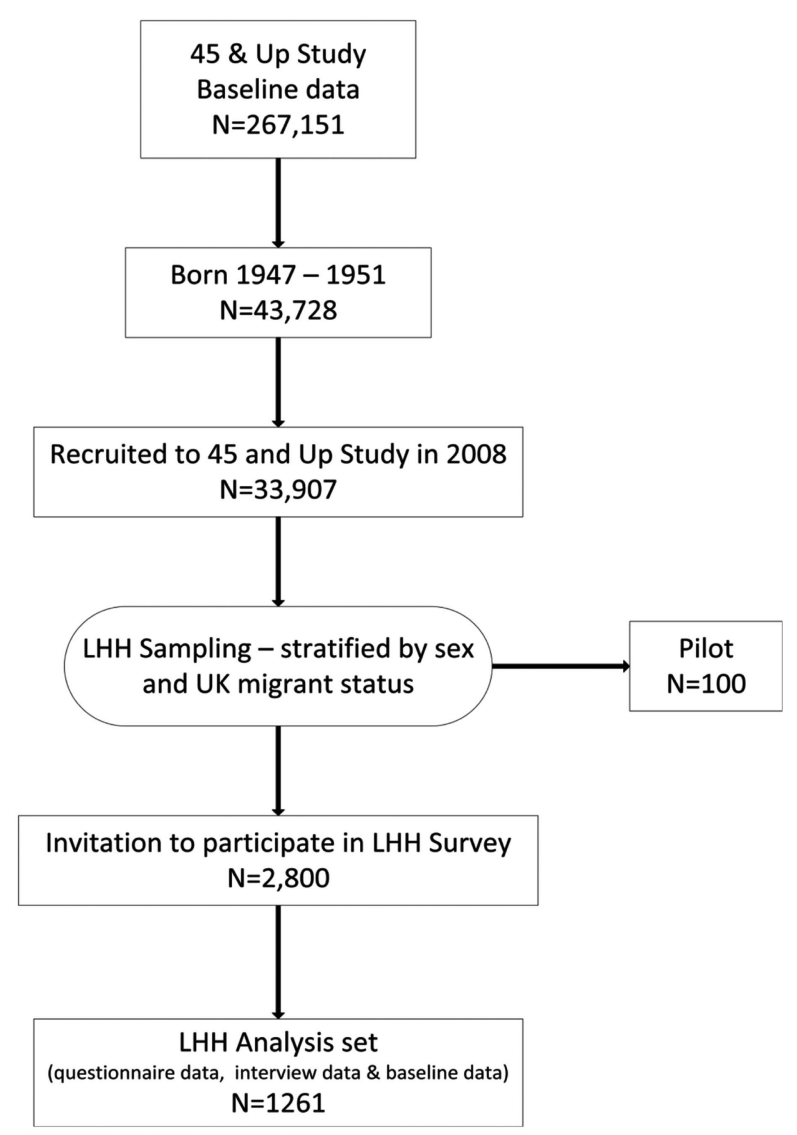

Figure 2 Flow diagram illustrating Life Histories and Health sample selection from within the 45 and Up Study framework.

questionnaire and participated in the follow-up telephone interview, reflecting an overall response rate of $45 \%$. Follow-up interviews took an average of $26 \mathrm{~min}$ to complete (range: $15-70 \mathrm{~min}$ ). Seventy participants had either lost or not completed their life history calendar when they were contacted for the follow-up interview. They were sent another life history calendar if needed and their interviews were rescheduled. There were 247 participants who completed the self-complete questionnaire, but did not participate in the follow-up interview. Compared with complete responders, the 247 participants who did not participate in the follow-up interview did not differ in current or childhood self-rated health, education, marital status or birth country (data not shown).

\section{Measures}

The measures used in the LHH project were largely based on those used in the ELSA life history interview ${ }^{27}$ and the baseline 45 and Up Study ${ }^{28}$ survey.

\section{Self-complete questionnaire}

In addition to standard demographics, the self-complete questionnaire included measures of childhood SES, relationship or bonding with parents, ${ }^{29}$ family composition and traumatic events across the life course. The self-complete questionnaire also contained measures of life-long health including birth weight, childhood hospital visits, long-standing illness and chronic disease across the life course, as well as measures relating to the participants' current status covering self-rated health, psychological health, ${ }^{30}$ limiting pain and physical mobility. Outcome measures such as life satisfaction, ${ }^{31}$ quality of life ${ }^{32}$ and unpaid productivity were also included.

\section{Telephone interview}

The follow-up telephone interview collected life history data about paid work, non-paid work, residential locations and personal relationships with survey participants' using their self-completed life history calendar as a memory aid. For each participant, every suburb (or country if overseas) they had lived in for 6 months or longer was recorded, with corresponding dates of residence. The participants also identified the number of years spent in personal relationships lasting 6 months or longer and the years in which these relationships occurred.

Paid work histories were given particular attention as these inform the measures of SES throughout adulthood. Details were recorded about each employment period lasting 6 months or longer, the sector (public vs private) and type (self-employed or not). The participants were asked whether each employment position involved heavy labour or physically demanding work, shift work, occupational hazards and whether they left the position due to poor health. Periods out of paid work were also noted, as well as the activities undertaken during this time (eg, caregiving). In addition, the participants identified which of their employment positions had made the 'most significant contribution to [their] financial resources-the one that helped [them] get ahead in life.' The participants were asked how many people (if any) they were responsible for and the level of education required for that position. The participants were also asked about their partner's current employment position.

\section{RESULTS}

\section{Participant characteristics}

Table 2 presents the characteristics of the LLH participants and comparisons with age-matched participants in the 45 and Up Study, and the general Australian population using 2011 census data where available. Employment status in the LHH Survey was consistent with Australian census data, but the LHH participants tended to report higher educational qualifications and better self-rated health compared with the Australian population. The distributions for gender and birth country were influenced by the sampling framework and are provided for context.

While there was some under-representation in terms of individuals with lower SES, as indicated by education and occupation, there were significant numbers of respondents across the full range of these variables for our analytical purposes. 
Table 2 General characteristics of the LHH Survey participants compared with 45 and Up Study participants and the Australian population aged $60-64$ years old

\begin{tabular}{|c|c|c|c|c|}
\hline & \multicolumn{2}{|c|}{$\begin{array}{l}\text { LHH Survey (60-64 years) } \\
\mathrm{N}=1261\end{array}$} & \multirow{2}{*}{$\begin{array}{l}45 \text { and Up } \\
\text { (60-64 years) } \\
\% \\
\end{array}$} & \multirow{2}{*}{$\begin{array}{l}\text { Australian census } \\
\text { data* }(60-64 \text { years) } \\
\%\end{array}$} \\
\hline & $\overline{\mathbf{N}}$ & $\%(95 \% \mathrm{Cl})$ & & \\
\hline \multicolumn{5}{|l|}{ Gender } \\
\hline Male & 684 & $54.2(51.5$ to 57.0$)$ & 46.7 & 49.6 \\
\hline Female & 577 & 45.8 (43.0 to 48.5$)$ & 53.3 & 50.4 \\
\hline \multicolumn{5}{|l|}{ Education } \\
\hline No school certificate & 18 & $1.4(0.8$ to 2.1$)$ & 10.6 & 17.9 \\
\hline Junior school certificate & 179 & $14.2(12.3$ to 16.1$)$ & 24.5 & 24.6 \\
\hline Senior school certificate & 106 & $8.4(6.9$ to 9.9$)$ & 9.8 & 12.4 \\
\hline Certificate or diploma & 592 & 46.9 (44.2 to 49.7$)$ & 26.9 & 28.2 \\
\hline Degree, masters or $\mathrm{PhD}$ & 366 & 29.0 (26.5 to 31.5$)$ & 22.8 & 16.9 \\
\hline \multicolumn{5}{|l|}{ Birth country $\dagger$} \\
\hline Australia & 865 & 68.6 (66.0 to 71.1$)$ & 73.1 & 64.4 \\
\hline UK & 274 & 21.7 (19.5 to 24.0$)$ & 11.1 & 10.2 \\
\hline Other & 122 & 9.7 (8.0 to 11.3$)$ & 15.0 & 25.4 \\
\hline \multicolumn{5}{|l|}{ Current marital status } \\
\hline Partnered (married/de facto) & 1028 & 81.5 (78.4 to 83.7$)$ & 78.6 & 73.7 \\
\hline Not partnered & 227 & $18.0(15.9$ to 20.1$)$ & 20.7 & 26.2 \\
\hline \multicolumn{5}{|l|}{ Self-rated health } \\
\hline Excellent & 190 & $15.1(13.1$ to 17.0$)$ & 16.0 & $16.9 \ddagger$ \\
\hline Very good & 518 & 41.1 (38.4 to 43.8$)$ & 38.1 & 33.4 \\
\hline Good & 390 & 30.9 (28.4 to 33.5$)$ & 30.5 & 30.9 \\
\hline Fair & 137 & $10.8(9.1$ to 12.6$)$ & 10.5 & 13.0 \\
\hline Poor & 24 & $1.9(1.1$ to 2.7$)$ & 1.9 & 5.8 \\
\hline Missing & 2 & $0.2(0.0$ to 3.8$)$ & 3.0 & - \\
\hline \multicolumn{5}{|l|}{ Current employment status } \\
\hline Full time paid work & 394 & 31.3 (28.7 to 33.8$)$ & 19.1 & 28.1 \\
\hline Part-time paid work & 220 & $17.4(15.4$ to 19.5$)$ & 14.9 & 18.1 \\
\hline Full-time and part-time paid work & 64 & 5.1 (3.9 to 6.3$)$ & 0.1 & - \\
\hline Not in paid work & 583 & 46.2 (43.5 to 49.0$)$ & 65.9 & 49.8 \\
\hline \multicolumn{5}{|l|}{ Current job type } \\
\hline Managers & 206 & 30.4 (26.9 to 33.8 ) & - & 15.5 \\
\hline Professionals & 196 & 28.9 (25.5 to 32.3$)$ & & 20.5 \\
\hline Trade people or technicians & 48 & 7.1 (5.1 to 9.0$)$ & & 11.9 \\
\hline Community or personal service & 32 & 4.7 (3.1 to 6.3$)$ & & 7.6 \\
\hline Clerical/administrative & 89 & 13.1 (10.6 to 15.7$)$ & & 16.3 \\
\hline Sales & 41 & $6.1(5.3$ to 7.8$)$ & & 7.3 \\
\hline Machinery driver and operators & 26 & 3.8 (2.4 to 5.3 ) & & 8.7 \\
\hline Labourers & 39 & 5.8 (4.0 to 7.5$)$ & & 10.3 \\
\hline Unable to code & 1 & 0.1 (0.0 to 0.4$)$ & & 0.2 \\
\hline
\end{tabular}

\section{Life course data summary}

Figure 3 provides a summary profile of the participant's responses for different life stages across multiple domains. In terms of childhood SES, there was heterogeneity in father's occupation: one-third of them stated that their mother was in paid work during their childhood, and most of the participants reported modest housing with a few amenities and shared bedrooms. On average, the participants held 6.7 jobs over their life course and have spent 37 years in paid work.
Family variables include early life information on parental bonds and childhood separation, and time-specific information on major life events including death of parents, adult relationships, children, divorce or separation, and other traumatic events. Many participants experienced traumatic events in childhood, with $37.7 \%$ reporting at least one traumatic event and 13\% experiencing separation from their mother prior to age 16 . In adult life, the participants reported an average of 1.4 married or de facto relationships with $37.8 \%$ having been divorced or separated. 


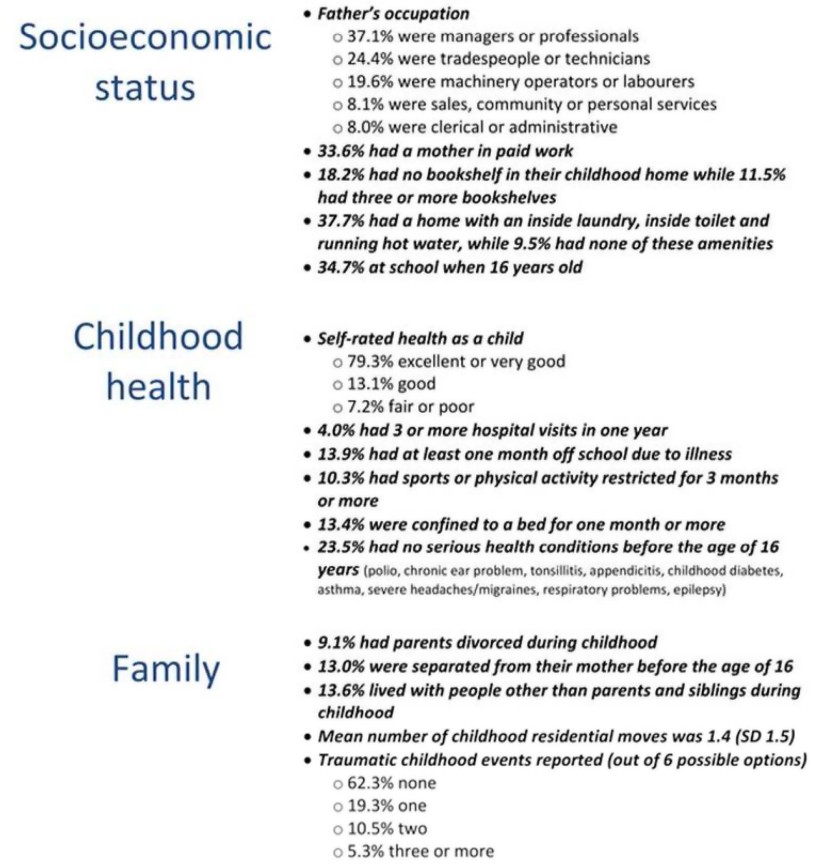

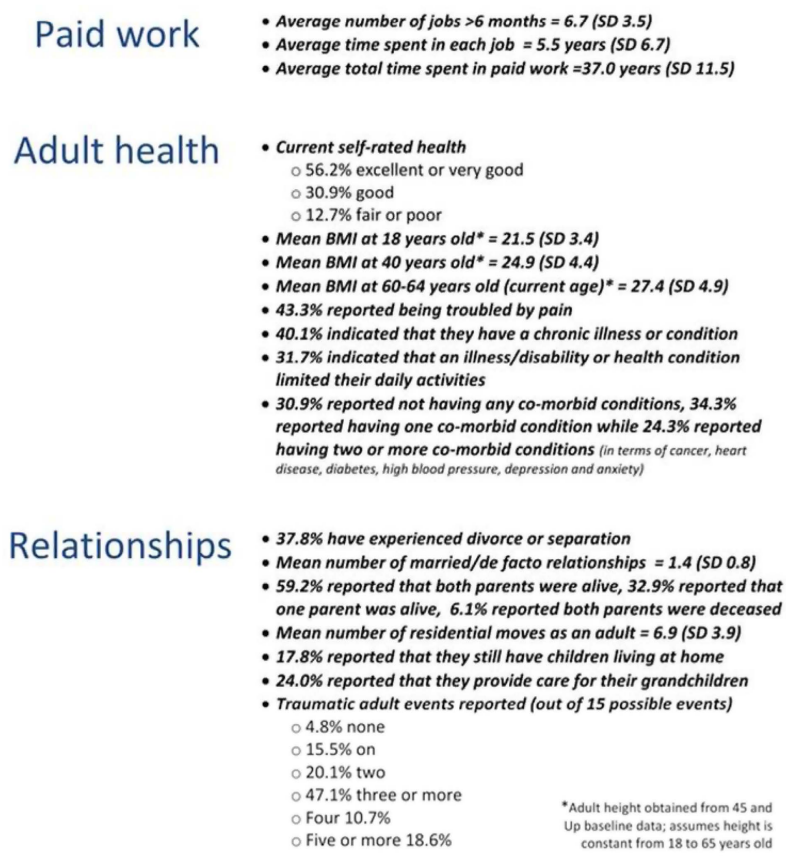

Figure 3 Summary profile of the life course data collected from the Life Histories and Health Survey participants ( $\mathrm{n}=1261$ ).

\section{Missing data}

Levels of missing data were generally relatively low and this was particularly apparent for work, health and accommodation histories, which are the key components of this study collected using the life history calendar and CATI combination. Certain items from the self-complete questionnaire had higher rates of missing data. For example, although $24 \%$ of participants reported experiencing severe financial hardship, only $64 \%$ of these people indicated the age at which the event was first experienced; this response pattern was similar for each traumatic event assessed. Health histories were also assessed in the self-complete questionnaire, but the data were more complete. For example, of the $9 \%$ of participants who had been diagnosed with heart disease, $86 \%$ recorded their age at diagnosis; and missing data were higher for weight at birth $(23 \%)$ than for current weight $(3.6 \%)$.

\section{Analysis strategy}

Planned analyses will identify how socioeconomic factors throughout life act independently, cumulatively and interactively to influence health and well-being outcomes in later life. ${ }^{33} 34$ Three types of hypothesised causal models, such as (1) critical period model; (2) sensitive period model and (3) accumulation model, will be analysed using a series of nested multiple regression analysis models. ${ }^{34}$ These models examine how socially patterned exposures and inequalities throughout the life course can influence health risks and socioeconomic position in late middle age. Social mobility will be used to describe the intrageneration and intergeneration variability in social status. ${ }^{35}$

As a second stage of the project, LHH and ELSA data will be harmonised to test hypotheses on how variation in the cross-national social, economic and policy contexts may have influenced exposures and outcomes earlier in life.

\section{DISCUSSION}

The LHH Survey was designed to collect detailed life history data to better understand the role of earlier life events on health and well-being outcomes for the early cohort of baby boomers in Australia. For many studies, the requirement of collecting useful data (eg, accurate, thorough and relevant) must be balanced against the logistics of data collection (eg, cost, convenience). Our study collected important information on the life course determinants of health in a relatively resource-efficient manner. The extensive pretesting and piloting resulted in relatively low levels of missing data and high interview completion rates in the main study. The sample characteristics of the main study were broadly comparable with the broader Australian population and covered a wide range of socioeconomic characteristics with some under-representation of lower education and non-English-speaking migrants. These factors indicate that the study was successful in adapting the ELSA life history module into a postal self-complete questionnaire 
and follow-up telephone interview to reach geographically dispersed participants.

The implementation of a pen-and-paper life history calendar that participants retained as a memory aid for the follow-up telephone interview was vital for the adapted data collection method used in our study. Rescheduling the follow-up interview if the participant had lost or not completed their life history calendar appeared to contribute to the high rate of completed interviews while also keeping the interview duration to an acceptable level. Throughout each stage of the survey development, the interviewers and participants acknowledged the importance of the life history calendar as a memory aid for gathering life history information. In addition, using the self-complete questionnaire also reduced the length of the telephone interview, reducing operational costs as well as participant interview fatigue.

The primary strength of this project is the richness of the data and resultant potential for answering complex research questions. For example, comparisons between those who spent their entire lives in Australia and England, and English migrants to Australia, will give us a unique opportunity to examine the influence of social structures and policy frameworks around health and wellbeing. Our group of English migrants will provide particularly valuable insights into how health is influenced by early life experiences (in their country of origin) in contrast with life exposures in Australia as their country of destination. Analyses addressing these and other key research questions are currently underway.

As a substudy of the ongoing 45 and Up Study, the LHH Survey participants may be followed over time to determine how earlier socioeconomic trajectories can influence health and well-being transitioning into older age. Incorporating baseline data from the 45 and Up Study also provides an opportunity to investigate a series of different research questions relating to life histories. The baseline data include alcohol and tobacco use, diet and physical activity levels, which may function as explanatory factors linking socioeconomic trajectories to health outcomes in later life.

Despite the relative heterogeneity and representativeness of the main study sample, a few limitations should be noted. Although the sample comprises participants from urban and rural communities, the study recruited residents from the state of NSW, who may not be representative of all Australians. Comparisons with the Australian census data indicate that our sample is healthier and more highly educated than the general population. Although differential response rates may induce a bias into prevalence estimates, Mealing et al $\mathrm{s}^{36}$ estimates of exposure-outcomes relationships remained consistent in the presence of contrasting response rates. While it is difficult to evaluate whether subgroups of participants found the survey more challenging to complete (eg, those with lower education, or those who do not speak English as their first language), patterns of missing data were similar across participants. Figure 3 shows a good variation in each of the study variables with differing experiences reported for life-long health and family structure across varying socioeconomic circumstances. The data collected on participants' work histories provide valuable information on SES at different time periods across the life course and allow for the assessment of social mobility over the life course and between generations.

This article has demonstrated the feasibility and value of life history data collection procedures and instruments that can economically increase the range of information collected on the socioeconomic context of ageing experiences over the life course. Systematic investigation of the varying temporal and societal context of ageing has the potential to widen understanding of the interplay between individual lives and changing social structures. This knowledge can inform action on the social determinants of health and optimal points in the life span and priority groups for social investments aimed at improving quality of life.

\section{Author affiliations}

${ }^{1}$ ARC Centre of Excellence in Population Ageing Research (CEPAR), Sydney, New South Wales, Australia

${ }^{2}$ Centre for Research on Ageing, Health, and Wellbeing, Research School of Population Health, Australian National University, Canberra, Australian Capital Territory, Australia

${ }^{3}$ Research Centre for Gender, Health and Ageing, School of Medicine and Public Health, Faculty of Health, University of Newcastle, Callaghan, New South Wales, Australia

${ }^{4}$ Ageing, Work and Health Research Unit, Faculty of Health Sciences, University of Sydney, Sydney, New South Wales, Australia

${ }^{5}$ School of Social Sciences, University of Manchester, Manchester, UK ${ }^{6}$ School of Population Health, University of Queensland, Herston, Queensland, Australia

Acknowledgements The authors thank Rosemary Cant, Rob Pedlow and the volunteer participants from National Seniors Australia and the Hunter Medical Research Institute (HMRI) for their involvement with the feasibility, pretest and pilot studies. This research was completed using data collected through the 45 and Up Study (http://www.saxinstitute.org.au). The 45 and Up Study is managed by the Sax Institute in collaboration with major partner Cancer Council NSW; and partners: the National Heart Foundation of Australia (NSW Division); NSW Ministry of Health; beyondblue; Ageing, Disability and Home Care, Department of Family and Community Services; the Australian Red Cross Blood Service; and UnitingCare Ageing. We thank the many thousands of people participating in the 45 and Up Study. The authors are also grateful for the generous help, cooperation and assistance received from Alison Cowle (The Sax Institute). Researchers from the Faculty of Health and Medicine at the University of Newcastle (JEB and PMF) are members of the Hunter Medical Research Institute (HMRI).

Contributors HK, JEB,KO, GM and JYN were responsible for the study inception and design. JN managed the field work, with data collection conducted by Crystal Clear Data for the self-complete questionnaires and Taverner Research McNair and Associates for the telephone interviews. PMF and VL were responsible for the amalgamation of the LHH data and statistical analysis. All the authors made contributions to conception and design, acquisition and management of data, or analysis and interpretation of data; were involved in the drafting the manuscript and/or provided critical revision for important intellectual content; and gave final approval of the manuscript to be published.

Funding Supported by an Australian Research Council Grant (DP 1096778, "Socio-economic determinants and health inequalities over the life-course: Australian and English comparisons") with investigators from the Universities 
of Sydney, Newcastle and Queensland (Australia) and the University of Manchester (UK).

Competing interests None.

Ethics approval The 45 and Up Study was approved by the University of New South Wales Human Research Ethics Committee. The LHH Survey was approved by the University of Sydney Human Research Ethics Committee (\#12744).

Provenance and peer review Not commissioned; externally peer reviewed.

Data sharing statement No additional data are available.

Open Access This is an Open Access article distributed in accordance with the Creative Commons Attribution Non Commercial (CC BY-NC 3.0) license, which permits others to distribute, remix, adapt, build upon this work noncommercially, and license their derivative works on different terms, provided the original work is properly cited and the use is non-commercial. See: http:// creativecommons.org/licenses/by-nc/3.0/

\section{REFERENCES}

1. Kendig H, Browning C. Directions for ageing well in a healthy Australia. Canberra, Australia: Academy of the Social Sciences in Australia, 2011:23-30.

2. Commission on Social Determinants of Health. Closing the gap in a generation: health equity through action on the social determinants of health. Final Report of the Commission on Social Determinants of Health. Geneva, Switzerland: World Health Organization, 2008.

3. Marmot M. The status syndrome: how social standing affects our health and longevity. New York, USA: Owl Books Henry Holt and Company, 2004.

4. Mishra GD, Ball K, Dobson AJ, et al. Which aspects of socio-economic status are related to health in mid-aged and older women? Int J Behav Med 2002;9:263-85.

5. Marmot M, Allen J, Bell R, et al. WHO European review of social determinants of health and the health divide. Lancet 2012;380:1011-29.

6. Australian Institute of Health and Welfare. Australia's health 2008. Cat no. AUS 99. Canberra: AlHW, 2008.

7. Marmot M. Social determinants of health inequalities. Lancet 2005;365:1099-104.

8. Blane $D$, Higgs $P$, Hyde $M$, et al. Life course influences on quality of life in early old age. Soc Sci Med 2004;58:2171-9.

9. Halleröd B, Gustafsson JE. A longitudinal analysis of the relationship between changes in socio-economic status and changes in health. Soc Sci Med 2011;72:116-23.

10. Britton A, Shipley M, Singh-Manoux A, et al. Successful ageing: the contribution of early-life and midlife risk factors. J Am Geriatr Soc 2008;56:1098-105.

11. Kendig $\mathrm{H}$, Browning $\mathrm{C}$, Teshuva $\mathrm{K}$. Health actions and social class among older Australians. Aust N Z J Public Health 1998;22:808-13.

12. Kuh D, Ben-Shlomo Y, Lynch J, et al. Life course epidemiology. J Epidemiol Community Health 2003;57:778-83.

13. Westermeyer J. Predictors and characteristics of successful ageing among men: a 48-year longitudinal study. Int J Aging Hum Dev 2013;76:323-45

14. Glasner T, van der Vaart W. Applications of calendar instruments in social surveys: a review. Qual Quant 2009;43:333-49.
15. Eerola M, Helske S. Statistical analysis of life history calendar data. Stat Methods Med Res 1 Nov 2012. Epub ahead of print. doi: 10.1177/0962280212461205

16. Belli RF. The structure of autobiographical memory and the event history calendar: Potential improvements in the quality of retrospective reports in surveys. Memory 1998;6:383-406.

17. Martyn KK, Belli RF. Retrospective data collection using event history calendars. Nurs Res 2002;51:270-4.

18. Belli RF, Smith LM, Andreski PM, et al. Methodological comparisons between CATI event history calendar and standardized conventional questionnaire instruments. Public Opin Q 2007;71:603-22.

19. Brückner E, Mayer KU. Collecting life history data: experiences from The German Life History Study. In: Giele JZ, Elder GH, eds. Methods of life course research: qualitative and quantitative approaches. Thousand Oaks: Sage, 1998:152-81.

20. Belli RF, Shay WL, Stafford FP. Event history calendars and question list surveys: a direct comparison of interviewing methods. Public Opin Q 2001;65:45-74.

21. Steptoe A, Breeze E, Banks J, et al. Cohort profile: the English Longitudinal Study of Ageing. Int J Epidemiol 2013;42:1640-8.

22. 45 and Up Study Collaborators. Cohort profile: the 45 and Up Study. Int J Epidemiol 2008;37:941-47.

23. Banks J, Breeze $\mathrm{E}$, Lessof $\mathrm{C}$, et al. Living in the 21st century: older people in England ELSA 2006 (Wave 3). London: The Institute for Fiscal Studies, 2008.

24. Blane D. Collecting retrospective data: development of a reliable method and a pilot study of its use. Soc Sci Med A 1996;42:751-57.

25. Belli R, Agrawal S, Bilgen I. Health status and disability comparisons between CATI calendar and conventional questionnaire instruments. Qual Quant 2012;46:813-28.

26. Australian Broadcasting Corporation. 80 days that changed our lives. Secondary 80 days that changed our lives. 2013. http://www.abc.net. au/archives/80days/timeline.htm

27. Ward K, Medina J, Mo M, et al. ELSA Wave Three: life history interview (a user guide to the data), version 1. National Centre for Social Research, 2009.

28. 45 \& Up Study. Study materials and publications. Secondary Study Materials and Publications, 2012. https://www.saxinstitute.org.au/ our-work/45-up-study/

29. Parker $\mathrm{G}$, Tupling $\mathrm{H}$, Brown LB. A parental bonding instrument. Br J Med Psychol 1979;52:1-10.

30. Radloff LS. The CES-D Scale: a self-report depression scale for research in the general population. Appl Psychol Meas 1977;1:385-401.

31. Diener E, Emmons RA, Larsen RJ, et al. The satisfaction with life scale. J Pers Assess 1985;49:71-5.

32. Wiggins RD, Netuveli G, Hyde M, et al. The evaluation of a self-enumerated scale of quality of life (CASP-19) in the context of research on ageing: a combination of exploratory and confirmatory approaches. Soc Indicators Res 2008;89:61-77.

33. Mishra GD, Chiesa F, Goodman A, et al. Socio-economic position over the life course and all-cause, and circulatory diseases mortality at age 50-87 years: results from a Swedish birth cohort. Eur J Epidemiol 2013;28:139-47.

34. Mishra G, Nitsch D, Black S, et al. A structured approach to modelling the effects of binary exposure variables over the life course. Int J Epidemiol 2008;38:528-37.

35. Denzin NK, Lincoln YS. The sage handbook of qualitative research. 3rd edn. Thousand Oaks, CA: Sage, 2005

36. Mealing N, Banks $\mathrm{E}$, Jorm $\mathrm{L}$, et al. Investigation of relative risk estimates from studies of the same population with contrasting response rates and designs. BMC Med Res Methodol 2010;10:26. 